Met abol i c moni tor i ng of advanced ut er i ne cervi cal cancer neoadj uvant chemot her apy by usi ng [ F- 18]- Fl uor odeoxygl ucose posi tron em ssi on t onogr aphy: pr el i mi nary resul t s i n three pati ents

\begin{tabular}{|c|c|}
\hline 著者 & $\begin{array}{l}\text { YOSH DA Yoshi o, KUROKAWA Tet suj i, KAWAHARA } \\
\text { Kazum, YAG HARA Aki ra, TSUCH DA Tat sur o, } \\
\text { OKAZAWA Hi dehi ko, FUJI BAYASH Yasuhi sa, } \\
\text { YONEKURA Yoshi har u, KOTSUJ Fum kazu }\end{array}$ \\
\hline $\begin{array}{l}\text { jour nal or } \\
\text { publ i cat } \mathrm{i} \text { on } \mathrm{ti} \mathrm{tl}\end{array}$ & Gynecol ogi c Oncol ogy \\
\hline vol une & 95 \\
\hline number & 3 \\
\hline page $r$ ange & $597-602$ \\
\hline year & 200412 \\
\hline URL & ht t p: //hdl . handl e. net /10098/1176 \\
\hline
\end{tabular}




\title{
Metabolic monitoring of advanced uterine cervical cancer neoadjuvant chemotherapy by using [F-18]-Fluorodeoxyglucose positron emission tomography: preliminary results in three patients
}

\author{
Yoshio Yoshida ${ }^{\mathrm{a}, *}$, Tetsuji Kurokawa ${ }^{\mathrm{a}}$, Kazumi Kawahara ${ }^{\mathrm{a}}$, Akira Yagihara ${ }^{\mathrm{a}}$, Tatsuro Tsuchida ${ }^{\mathrm{b}}$, \\ Hidehiko Okazawa ${ }^{c}$, Yasuhisa Fujibayashic, Yoshiharu Yonekura ${ }^{c}$, Fumikazu Kotsuji ${ }^{a}$ \\ "Depariment of Obstetrics and Gynecology, University of Fukui, Fukui-ken 910-1103. Japan \\ ${ }^{\text {t}}$ Department of Radiology and Neurosurgery, University of Fukui, Fukui-ken 910-1103, Japan \\ ${ }^{\mathrm{c}}$ Biomedical Imaging Research Center, University of Fukui, Fukui-ken 910-1103, Japan
}

\begin{abstract}
Objective. The aim of this report is to describe the potential clinical utility of tracer [F-18]-Fluorodeoxyglucose (FDG) uptake, quantitated as a standardized uptake value (SUV) by positron emission tomography (PET), to evaluate treatment response to neoadjuvant chemotherapy (NAC) in advanced uterine cervical cancer.

Methods. We briefly describe the clinical courses of three women with advanced cervical cancer who were treated with neoadjuvant chemotherapy (NAC) prior to radical hysterectomy and who were analyzed for correlation with the decrease in tumor volume by magnetic resonance imaging (MRI), in SUV by FDG-PET, and by histologic response.

Results. In these individuals, tumor volume and SUV were decreased by NAC. The decrease in SUV by FDG-PET was better correlated to histologic response for NAC than MRI was in advanced cervical cancer.

Conclusions. Measurement of SUV by FDG-PET has clinical utility in evaluating treatment response for NAC in advanced cervical cancer. Although work in this field is still in the early stages, this report demonstrates that SUV by FDG-PET has the potential to become the new standard for monitoring the treatment response of NAC in cervical cancer. This monitoring approach must be proven in a larger number of patients for both primary and secondary lesions and should be further explored in another gynecologic cancer.

(c) 2004 Elsevier Inc. All rights reserved.
\end{abstract}

Keywords: FDG-PET for monitoring treatment response; Cervical cancer

\section{Introduction}

For many years, the standard approach to determining whether a tumor is sensitive or resistant to treatment has relied on various anatomic imaging techniques, such as computed tomography $(\mathrm{CT})$ and magnetic resonance imaging (MRI) [1]. These techniques have a number of limitations, however, because they evaluate only the size

* Corresponding author. Department of Obstetrics and Gynecology, University of Fukui, Matsuoka-Cho, Yoshida-Gun, Fukui-ken 910-1103, Japan. Fax: +81776618117.

E-mail address: yyoshida(a) fimsrsa.fukui-med.ac.jp (Y. Yoshida). of a lesion, not its viability, proliferative rate, or physiologic state.

Positron emission tomography (PET) with tracer [F-18]Fluorodeoxyglucose (FDG) (FDG-PET) is a unique, noninvasive method for studying biochemical and metabolic changes in cancer tissue [2]. Although work in this field is still in the early stages, studies are already demonstrating that it is becoming the new standard for some tumors and treatments. However, few studies monitoring the response treatment of gynecologic cancer have been done with FDG tracers [3-5], and there has been no comparison of the response determined by MRI, FDG-PET, and histopathological studies before and after chemotherapy. 
We briefly describe the clinical courses of three women with advanced cervical cancer who were treated with neoadjuvant chemotherapy (NAC) prior to radical hysterectomy. The decrease in tumor volume was compared by magnetic resonance imaging (MRI), in standardized uptake value (SUV) by FDG-PET, and by histologic response to chemotherapy.

\section{Materials and methods}

A prospective analysis was conducted on patients with advanced cervical cancer evaluated at our institution from 2002 to 2003 . The three patients with primary, previously untreated, histologically confirmed invasive squamous cell carcinoma included were 51,59 , and 72 years old. After the diagnosis of cervical cancer, all of them were treated with neoadjuvant chemotherapy (NAC) prior to radical hysterectomy. For each patient, comparisons were made of the assessment of response determined by MRI, FDG-PET studies, and histopathological studies before and after NAC.

\section{Chemotherapy protocol}

Neoadjuvant chemotherapy was done according to the following schedule: $25 \mathrm{mg} / \mathrm{m}^{2}$ per day CDDP in $1000 \mathrm{ml}$ of physiological saline and $1000 \mathrm{mg} / \mathrm{m}^{2}$ per day $5-\mathrm{FU}$ in $1000 \mathrm{ml}$ of glucosaline solution were administered continuously through continuous perfusion for 4 days according to our published Phase I study [6]. All patients received hydration before and after the procedure. Diuretics and antiemetics were administrated as necessary. Two treatment cycles every 4 weeks were performed, based on the results of our published Phase I study [6].

\section{Magnetic resonance imaging}

MRI was performed using a 1.5-T system (Signa; GE Medical Systems). The pelvic imaging protocol utilized included axial and sagittal T1-weighted gradient-echo sequences (TR/TE, 89.3/4.1) with the administration of an IV contrast medium. Intravenous gadolinium diethylenetriamine pentaacetic acid (Magnevist; Schering, Tokyo, Japan) was administered at the usual dose of $0.1 \mathrm{mmol} / \mathrm{kg}$ of body weight. In addition, axial and sagittal T2weighted turbo-spin-echo sequences (2800/138) were performed [7].

\section{PET procedures}

The three patients underwent PET scanning with a whole-body tomography scanner (Advance; General Electric Medical System, Milwaukee, WI) with continuous bladder irrigation. FDG was produced using a small cyclotron (OSCAR3; Oxford Instruments, Oxford, UK) and an automated synthesis system (NKK, Tokyo, Japan).
The patients fasted for at least $12 \mathrm{~h}$ before tracer administration, and approximately $185 \mathrm{MBq}$ of FDG was injected intravenously. Whole-body emission scanning was started $40 \mathrm{~min}$ after FDG administration, and PET data were acquired for $12-14 \mathrm{~min}$ with $6-7$ bed positions [7-10]. PET findings for the transaxial, coronal, and sagittal sections were interpreted by two experienced nuclear medicine physicians (H.O. and T.K.).

\section{Image analysis}

The PET data were reconstructed using the iterative reconstruction algorithm and segmented attenuation correction (IR/SAC) method, and were resliced into transaxial, coronal, and sagittal sections with the grey scale in the standardized uptake value (SUV) calculated by the normalization of the regional radioactivity concentration to injected dose and body weight. If regions of FDG accumulation were manifested on the FDG-PET images, the site of each region was evaluated. The estimation of lesions on the FDG-PET images was based on visual correlation with MRI, slice by slice. When tissue samples were obtained at laparotomy, the lesions were evaluated according to MRI findings [10]. Hypermetabolic lesions that were more intense than physiologic liver uptake were considered positive for malignancy. In the liver, lesions cannot be attributed to structures that physiologically accumulate FDG, such as the bladder, the ureters, or the gastrointestinal tract $[9,10]$.

For quantitative evaluation of regional radioactivity using static FDG-PET images, circular regions of interest (ROIs) $3 \mathrm{~mm}$ in diameter were placed manually over the hypermetabolic lesions in the primary and metastatic lesions by one nuclear medicine physician (H.O.). SUVs were obtained from three areas of high radioactivity accumulation as visualized in the primary and metastatic lesions, and the mean SUV was calculated for each tumor. For statistical analysis, mean SUVs were used [10].

\section{Pre-and post-treatment tumor measurements}

The response to neoadjuvant chemotherapy was analyzed on abdominal MRI using the published Response Evaluation Criteria in Solid Tumors (RECIST) criteria [11]. Surgery was then performed in patients showing a tumor response.

\section{Histopathological grading}

Finally, after surgery, the results of the two methods (MRI and FDG-PET) were compared with the histological evaluation of the effects of neoadjuvant chemotherapy using the surgical specimens according to the method of Shimosato et al. [12] (Table 1). In FDG-PET, the pretreatment SUV and posttreatment SUV were compared with the histologic evaluation of residual tumor cells. In 
Table 1

Criteria for histologic evaluation of effects of radiotherapy and chemotherapy according to Shimamoto et al.

$0 \quad$ No recognized changed in tumors.

1 Minimal cellular changes presents, but a majority of tumor cells appear viablc.

2a Despite the presence of cellular changes and partial destruction of the fumor, the tumor is still readily recognizable, and a good number of tumor cells appear viable.

$2 \mathrm{~b}$ The tumor destruction is extensive, but viable cell nests are present in small areas of the tumor (<one-quarter of the tumor mass, excluding areas of coagulation necrosis).

3 Only a few scattered, markedly altered, presumable non viable tumor cells are present singly or in small clusters; viable cells are hardly encountered.

$4 \quad$ No tumor cells remain in any section.

addition, to evaluate treatment effect, the size of the tumor was determined by measuring the maximum area in a single transaxial section of the contrast-enhanced MRI $[11,13]$.

\section{Results}

Patient 1 is a 51-year-old woman who was diagnosed with stage $\mathrm{Ilb}$ advanced cervical cancer according to the criteria of the International Federation of Gynecology and Obstetrics [6]. Pelvic MRI confirmed the presence of a $4.0 \times 4.0 \mathrm{~cm}$ (maximum diameter in the cross-sectional area) cervical mass, which had typical features of cervical cancer as it presented with a high-signal-intensity mass on a T2-weighted MR image. This lesion was shown to be highly metabolically active by FDG-PET $(\mathrm{SUV}=6.1)$. MRI and FDG-PET did not reveal other sites of suspected metastatic lesions. After the patient received two-cycle neo-adjuvant chemotherapy, the cervical lesion obviously decreased in size $(0.5 \times 0.5)$ as shown on MRI and in SUV (0.9) on FDG-PET. After neo-adjuvant chemotherapy, the patient underwent radical hysterectomy. Histological examination of the surgical specimen in the cervical lesion showed some viable tissues surrounding a necrotic region, thus suggesting a complete response to treatment of the cervical lesion, as shown by MRI and FDG-PET study.

Patient 2 is a 59 -year-old woman who was diagnosed with stage Illb advanced cervical cancer according to the criteria of FIGO. Pelvic MRI confirmed the presence of a $5.5 \times 4.5 \mathrm{~cm}$ cervical mass, a $3.0 \times 1.5 \mathrm{~cm}$ left external iliac enlarged lymph node (LN), and a $1.0 \times 1.0 \mathrm{~cm}$ left common iliac enlarged LN. The primary cervical lesion was shown to be highly metabolically active by FDG-PET $($ SUV $=9.0)$. Similarly, the presence of increased uptake both in the left external iliac LN (SUV = 4.7) and in the left common iliac LN (SUV $=2.7$ ) was identified with FDG-PET. After two cycles of neo-adjuvant chemotherapy, MRI and FDG-PET studies were repeated. MRI showed a significant volume reduction in the cervical lesion and the left common iliac LN (diameters reduced from $5.5 \times 4.5$ to $0.9 \times 0.9$ and from $1.0 \times 1.0$ to less than $0.5 \mathrm{~cm}$, respectively) but did not show a significant volume reduction in the left external iliac LN (diameter reduced from $3.0 \times 1.5$ to $2.5 \times 1.2 \mathrm{~cm}$ ) (Figs. $1 \mathrm{~A}$ and $\mathrm{B}$ ). However, the FDG-PET study showed a significant metabolic activity reduction in all lesions (metabolic activity reduced from 9.0 to 2.1 in the primary lesion, from 4.7 to 2.3 in the left external iliac LN, and from 2.7 to undetectable in the left common iliac LN) (Figs. $1 \mathrm{C}$ and D). Histological examination of the surgical specimen showed only a few viable cancer cells in the primary lesion and no viable cancer cells in the common iliac LN. In the left external iliac LN, which had a disparity in volume reduction on $\mathrm{MRI}$ and in metabolic activity reduction on FDG-PET, few viable cancer cells surrounding a necrotic region were seen. Criteria for histological evaluation of effects of chemotherapy according to Shimosato et al. [12] were grade $2 b$ in the left external iliac LN (Fig. 1E). Thus, in this case, measurement of SUV in FDG-PET had a better correlation to histologic response for NAC than MRI did in advanced cervical cancer.

Patient 3 is a 72-year-old woman who was diagnosed with stage IIIb advanced cervical cancer according to FIGO. Pelvic MRI confirmed the presence of a $4.5 \times$ $4.0 \mathrm{~cm}$ (maximum diameter in MRI) cervical mass. This lesion was shown to be highly metabolically active by FDG-PET (SUV $=10.1$ ). MRI and FDG-PET did not reveal other sites of suspected metastatic lesions. After the patient received two-cycle neoadjuvant chemotherapy, the cervical lesion showed a $100 \%$ reduction in size on MRI (Figs. 2A and B). However, the FDG-PET study was still positive for a primary cervical (SUV $=6.7$ ) lesion (Figs. $2 \mathrm{C}$ and $\mathrm{D})$. Response to neoadjuvant chemotherapy was evaluated by abdominal MRI using the RECIST criteria [11]. A complete response was observed in patient 3. After neo-adjuvant chemotherapy, the patient underwent radical hysterectomy. Histological examination of the surgical specimen showed many viable cancer cells in the primary lesion. Criteria for histological evaluation of effects of chemotherapy according to Shimosato et al. [12] was grade $2 \mathrm{a}$ in the primary (Fig. 2E). Thus, in this case, measurement of SUV in FDG-PET had a better correlation to histologic response for NAC than MRI did in advanced cervical cancer.

All three primary, histologically confirmed invasive squamous cell carcinoma lesions were shown to be highly metabolically active by FDG-PET (SUV $=6.1,9.0$, and 10.1, respectively). All patients were evaluable using RECIST critcria [11]. Patient $1(\mathrm{SUV}=6.1)$ and patient 2 $(\mathrm{SUV}=9.0)$ were partial responders, and patient $3(\mathrm{SUV}=$ 10.1) was a complete responder. In the three patients, the primary uptake of FDG (evaluated using SUV) did not correlate with response (evaluated using RECIST) to treatment. And all three patients have had progression-free 
A

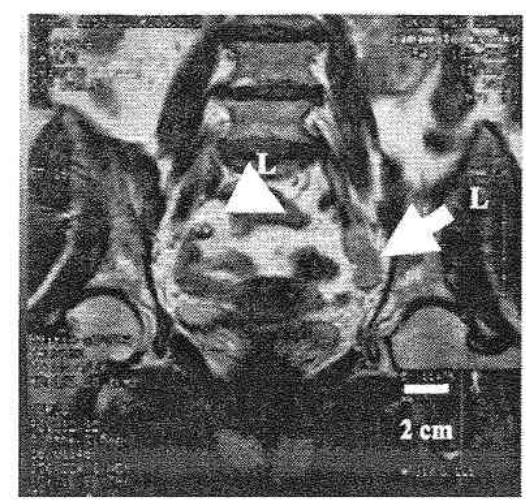

C

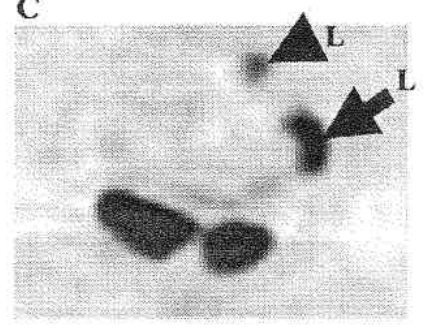

B

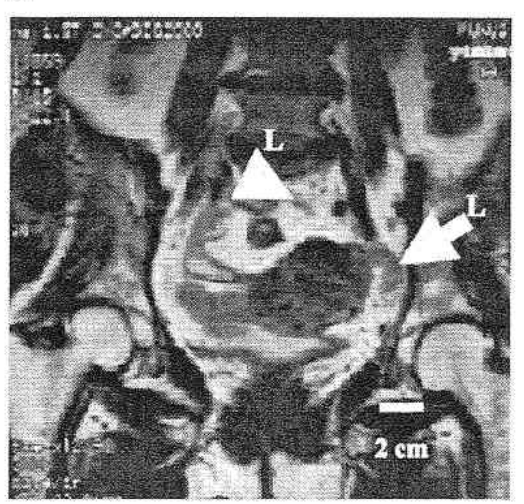

D

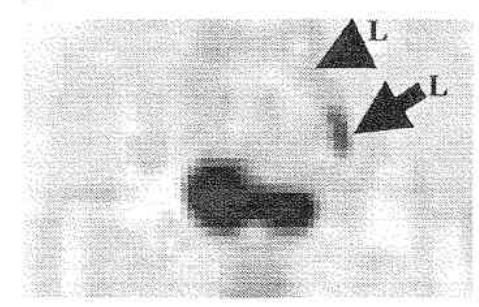

$\mathbf{E}$

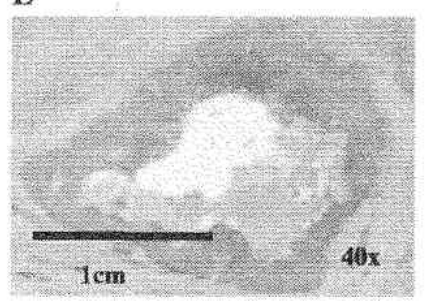

Fig. 1. Case 2: Stage IIIb advanced cervical cancer with left external iliac enlarged lymph node (LN)(L-arrow) and a left common iliac enlarged LN (L, arrow head). Pelvic MRI that was taken before (A) and after (B) treatment with neoadjuvant chemotherapy showed a significant volume reduction in the left common iliac LN (L, arrow head) (diameters reduced from $1.0 \times 1.0$ to less than $0.5 \mathrm{~cm}$ ) but did not show a significant volume reduction in the left external iliac LN (L, arrow) (diameter reduced from $3.0 \times 1.5$ to $2.5 \times 1.2 \mathrm{~cm}$ ). In FDG-PET images, SUV calculated before (C) and after (D) treatment showed a significant metabolic activity reduction in all lesions (arrows) (metabolic activity reduced from 4.7 to 2.3 in the left external iliac LN (L, arrow), and from 2.7 to undetectable ( $\mathrm{L}$, arrow head) in the common iliac LN). (E) In the right external iliac LN, which had a disparity in volume reduction on MRI and in metabolic activity reduction on FDG-PET, few viable cancer cells surrounding a necrotic region were seen. Criteria for histological evaluation of effects of chemotherapy according to Shimosato et al. was grade $2 b$ in the right external iliac LN (arrow) (original magnification, $40 \times$, hematoxylin staining).

survival $(22,18$, and 12 months for cases 1,2 , and 3 , respectively).

\section{Discussion}

This study clearly indicates the superiority of FDG-PET to MRI in monitoring response to chemotherapy treatment. Thus, FDG-PET is an important tool for knowing whether treatment should continue and when to discontinue chemotherapy for cervical cancer.

Clinical imaging of many types, including X-ray, nuclear, and ultrasound techniques, is widely used to detect and stage cancer. After that is accomplished, the appropriate therapy must be chosen, and this may include a combination of surgery, radiation, chemotherapy, and biologic therapies [1]. Evaluating the success of such treatment often requires additional imaging studies. Although cross-sectional images obtained with CT and MRI have become standard in evaluating treatment response, they create a number of issues. The major issues that affect the sensitivity and specificity of detecting response by measuring size include delays in shrinkage of dying tumors, slow growth of tumors despite unsuccessful treatment, and the persistence of fibrotic or necrotic tumors.

Over the past several years, studies of lymphoma [14,15], brain tumor [15], colorectal cancer [15], and lung cancer [16] have clearly indicated the superiority of FDG-PET to cross-sectional images, such as $\mathrm{CT}$, in monitoring response to treatment. However, few studies of gynecologic cancer [3-5] in monitoring response treatment have been done with FDG tracers. One of the major reasons for this is that FDG has been somewhat limited in its use in gynecologic disease due to the difficulty in detecting tumors adjacent to the 
A

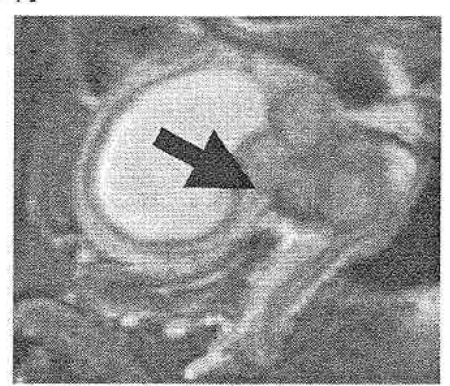

C

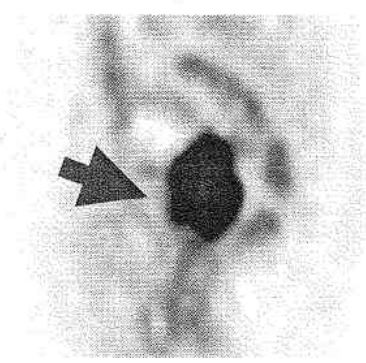

B

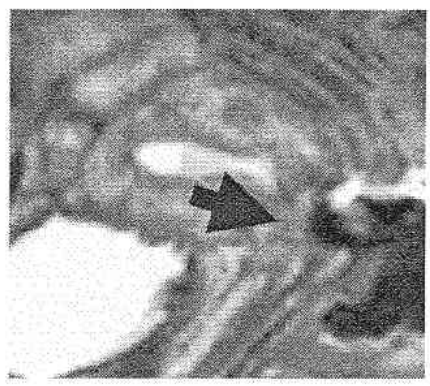

D

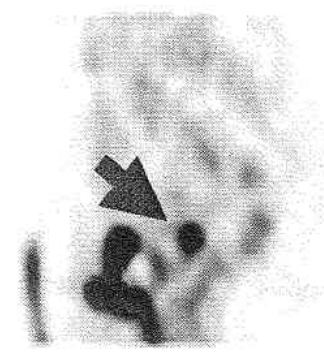

E

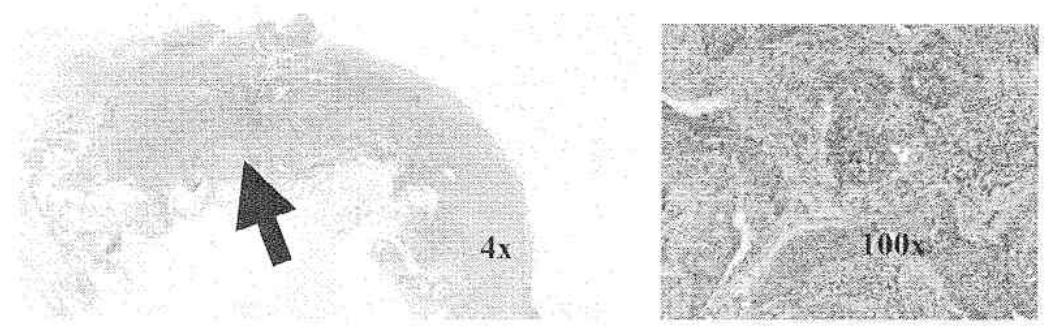

Fig. 2. Case 3: Stage IIIb advanced cervical cancer. Pelvic MRI that was taken before (A) and after (B) treatment with neoadjuvant chemotherapy showed a significant volume reduction in cervical lesion (arrows) (diameters reduced from $4.5 \times 4.0$ to undetectable). In the FDG-PET images, SUV calculated before (C) and after (D) treatment did not show metabolic activity reduction in cervical lesion (arrows) (metabolic activity reduced from 10.1 to 6.7). (E) In the primary lesion, which had a disparity in volume reduction on MRI and in metabolic activity reduction on FDG-PET, many viable cancer cells were seen. Criteria for histological evaluation of effects of chemotherapy according to Shimosato et al. were grade 2a in the primary (arrow) (original magnification, left: $4 \times$, right: $100 \times$, hematoxylin staining).

bladder and bowel. To overcome this limitation, in this study, FDG-PET with continuous bladder irrigation and at least 12-h fasting for patients before tracer administration was used for evaluating cervical cancer [7-10].

In three patients with advanced cervical cancer presented in this report, we have demonstrated the supcriority of FDGPET to MRI in monitoring the response of NAC in advanced cervical cancer in a histological study. A principal finding of this work is that SUV of FDG-PET is strongly correlated with pathological findings obtained from surgical specimens in both the primary and lymph node status. As shown here, anatomic imaging techniques evaluate only the size of a lesion, not its viability, proliferative rate, or physiologic state. Thus, actually determining whether this treatment is successful and whether it should continue should be dependent on the evaluation of both anatomical imaging and FDG-PET. Knowing when to discontinue therapy should also be dependent on both techniques because treatments are toxic and very costly.
It is also important to note that the timing of response is certainly the most common issue in the assessment of response chemotherapy. The standard approach for patients receiving cytotoxic drugs is to have CT or MRI done every 2 months [17]. Even when treatment is successful, it can take months to become evident in CT or MRI, and it is difficult to differentiate cases with slowly growing tumors in which treatment is not helping from those in which the treatment has induced a decrease in the tumor growth rate. In this study, we used PET at the end of a course of treatment, 2 months after the first treatment. For this report, it was considered appropriate that the timing of the response was at the end of treatment. When evaluating response to therapy, we quantitated the imaging results with a semiquantitative evaluation using SUV. If the SUV was still high, another treatment was considered because failed primary treatment is critical and carries the worst prognosis. In this report, it was considered that malignant lesions had a value of more than 2.0 [9]. 
Based on this experience, in primary cervical cancer, measurement of treatment by FDG-PET may become more important as more successful treatment options become available. It is important for this imaging approach to be evaluated in another gynecologic cancer.

\section{Acknowledgments}

This work was supported by a Grant-in-Aid for Exploratory Research (15790881) from the Ministry of Education, Culture, Sports, Science and Technology of Japan and by a Grant-in-Aid for Scientific Research C (14571549) from the Japan Society for the Promotion of Science. We thank Dr. Yoshiaki Imamura for evaluation of pathology, and Fumiko Maki and Makoto Oota for excellent technical assistance. And we gratefully thank Nobuyo Matui for assistance with manuscript preparation.

\section{References}

[1] Asher SM, Imaoka I, Hricak H: Diagnostic imaging techniques in gynecologic oncology. In: William JH, Carlos AP, Robert CY, editors. Principles and practice of gynecologic oncology, third ed. Philadelphia: Lippincott Williams \& Wilkins, 1999. p. 629-68.

[2] Gambhir SS. Molecular imaging of cancer with positron emission tomography. Nat Rcv, Cancer 2002;2:683-93.

[3] Grigsby PW, Sicgel BA, Dehdashti F, Mutch DG. Posttherapy surveillance monitoring of cervical cancer by FDG-PET. Int J Radiat Oncol Biol Phys 2003;55:907-13.

[4] Dose J, Hemminger GE, Bohuslavizki KH. Therapy monitoring using FDG-PET in metastatic cervical cancer. Lancet Oncol 2000;1:106.

[5] Baum RP, Przetak C. Evaluation of therapy response in breast and ovarian cancer patients by positron emission tomography (PET). Q J Nucl Med 2001;45:257 68 .

[6] Yoshida Y, Goto K, Kawahara K, Kurokawa T, Shukunami K, Kotsuji F. A phase I study of continuous administration of 5-Fluorouracil/ cisplatin in advanced uterine cervical cancer. Anticancer Res 2002;22:3473-6.

[7] Kawahara K, Yoshida Y, Kurokawa T, Suzuki Y, Nagahara K, Tsuchida T; et al. Evaluation of positron emission tomography with tracer [18] -Fluorodeoxyglucose in addition to magnetic resonance imaging in the diagnosis of ovarian cancer in selected women after ultrasonography. J Comput Assist Tomogr 2004; 28:505-16.

[8] Kurokawa T, Yoshida Y, Kawahara K, Tsuchida T, Fujibayashi Y, Yonekura Y, et al. Whole-body PET with FDG is useful for following up an ovarian cancer patient with only rising CA125 levels within the normal range. Ann Nucl Med 2002;16:491-3.

[9] Yoshida Y, Kurokawa T, Kawahara K, Tsuchida T, Okazawa H, Fujibayashi $Y$, et al. Incremental benefits of positron emission with [F18] fluorodeoxiglucose over computed tomography alone for the preoperative staging of ovarian cancer. AJR, Am J Roentgenol 2004; $182: 227-33$.

[10] Kurokawa T, Yoshida Y, Kawahara K, Tsuchida T, Okazawa H, Fujibayashi Y, et al. Expression of GLUT-1 glucose transfer, cellular proliferation activity and grade of tumor correlate with [F-18]fluorodeoxyglucose uptake by positron emission tomography in epithelial tumors of the ovary. Int J Cancer 2004;109:926-32.

[11] Therasse P, Arbuck SG, Eisenhauer EA, Wanders J, Kaplan RS, Rubinstein L, et al. New guidelines to cvaluate the response to treatment in solid tumors. European Organization for Research and Treatment of Cancer, National Cancer Institute of the United States, National Cancer Institute of Canada. J Natl Cancer Inst 2000;92: 205-216.

[12] Shimosato Y, Oboshi S, Baba K. Histological evaluation of effects of radiotherapy and chemotherapy for carcinomas. Jpn J Clin Oncol 1971; $1: 19-35$.

[13] Kitagawa Y, Sadato N, Azuma H, Ogasawara T, Yoshida M, Ishii Y, et al. FDG PET to evaluate combined intra-arterial chemotherapy and radiotherapy of head and neck neoplasms. J Nucl Med 1999;40: $1132-1137$

[14] Schoder H, Meta J, Yap C, Ariannejad M, Rao J, Phelps ME, et al Effect of whole-body (18)F-FDG PET imaging on clinical staging and management of patients with malignant lymphoma. J Nucl Med 2001 ; 42:1139-43.

[15] Delbeke D. Oncological applications of FDG PET imaging: brain tumors, colorectal cancer, lymphoma and melanoma. J Nucl Med 1999;40:591-603.

[16] Vansteenkiste JF, Stroobants SG. Positron emission tomography in the management of non-small cell lung cancer. Hematol Oncol Clin North Am 2004;18:269-88.

[17] Hockstra CJ, Paglianiti I, Hockstra OS, Smit EF, Postmus PE, Tcule AA, Lammertsma AA. Monitoring response to therapy in cancer using [18F]-2-Fluoro-2-deoxy-D-glucose and positron emission tomography: an overview of different analytical methods. Eur J Nucl Med 2000;27:731-43. 